\title{
Two Tier Approach for Automatic Retrieval of MRI Brain Image by Feature Extraction
}

\author{
Gayatri Chavan \\ ME Scholar \\ Pimpri Chinchwad College of Engineering \\ Pune-44
}

\author{
Sonal Gore \\ Assistant Professor \\ Pimpri Chinchwad College of Engineering \\ Pune-44
}

\begin{abstract}
Content Based Image Retrieval(CBIR) is also known as Query By Image Content(QBIC) is the one of the application of computer vision techniques, which retrieves the images from the image database instead of text. The CBIR is gaining the popularity in medical domain, because CBIR techniques help to search the digital images in the large database. The proper feature extraction and matching process, retrieves stored image from database by supplying the query image. The features such as color, shape texture or combination of them. In this paper, focused on texture and shape feature for extracting the image from database, by introducing the SVM (support vector machine) classifier followed by KNN (Knearest neighbor).

In this paper propose a efficient retrieval of image using a supervised classifier which focused on the texture features. Segmentation based Fractal Texture Analysis or SFTA algorithm is used to extract the texture feature from images. To select best features from extracted features to train the classifier, achieve better feature optimization. The classification is done on the database and it is classified in to three categories such as normal, benign and malignant. The query image is classified using a classifier and retrieve the relevant image from the database from a particular class.
\end{abstract}

\section{Keywords}

Content Based Image Retrieval, Feature extraction, MRI brain tumor image, SVM

\section{INTRODUCTION}

Medical imaging is the most important techniques and processes used to create the human anatomy image, which are used for clinical research and education, diagnostics and planning treatment .CBIR is most important research areas in medical image processing field recently. In the medical field Magnetic Resonance imaging(MRI), ultrasound(US), endoscopy, microscopy,colonos copy,computed tomography(CT) are stored as a digital images, which further used for diagnostics, research or treatment are produced centers ever increasingly. With the increasing growth of population ratio, cancer has become a global public health problem. In the worldwide every year,12.7 million public are diagnosed with cancer and 7.6 million people died of cancer[SVM_MRI].A brain tumor is the growth of abnormal cells in the tissues of the brain. Brain tumor can be benign (noncancerous) or malignant (cancerous).

The methodology includes following modules: Image preprocessing, feature extraction, feature selection, training \& classification/testing.

To get the better quality of images, preprocessing is performed on images. Medical images are degraded by different type of noise which affects the accuracy of result. It is very important to have good quality of image for accurate observation for the given application. Image retrieval is performed on the MR images using method which combined texture and shape feature of the image. The other major task is doing the extensive analysis of the database structure using clustering algorithm. The segmentation based fractal texture analysis is used for texture feature extraction, based on the evolution of the texture similarity to build the knowledge base.

In this paper has shown that the classification is perform on the MR images related to human brain have classified supervised technique in support vector machine followed by $\mathrm{K}$-nearest neighbor (K-NN) also group pixel based on their similarities in each feature image can be used to classify the MR images. Here suggest to use segmentation based fractal texture analysis to extract the texture feature which works much faster in terms of feature extraction time, when compared to the Gabor and Haralick methods. After feature extraction, feature selection has been applied to reduce the dimensionality of data which directly reduces the computation cost. In classification part the MRI brain tumor images are classify into three categories such as normal, benign, and malignant by using support vector machine as a supervised machine technique. To calculate weighted score and similarity matching compare the precision and recall to improve the accuracy of retrieval image.

A general flowchart of the proposed CBIR is shown in Figure 1. It shows exact steps of the CBIR system which carried out for image retrieval of the MR images from database, e.g., Feature extraction, feature selection, major type classification (first tire), and image retrieval according to the subtypes using KNN (second tire).

A general flowchart of the proposed CBIR is shown in Figure 1. It shows exact steps of the CBIR system which carried out for image retrieval of the MR images from database, e.g., Feature extraction, feature selection, major type classification (first tire), and image retrieval according to the subtypes using $\mathrm{KNN}$ (second tire).

\section{LITERATURE SURVEY}

1. Guoyong Duan [1] , this paper shows the advantages of the content based image retrieval system, as well as technologies. Also introduces all the feature extraction methods such as color, texture shape for image retrieval with its advantage.

2. Priyanka P. Buch[2] ,this paper gives the comparative analysis of the content based image retrieval using both color and texture. They have used two different approaches for the texture exraction.the first approach is using Gabor filter for texture extraction. Second approach is using Gabor filter for texture extraction. 
3. Ketan Machhale[3],this paper shows the research work which involves the SVM and SVM-KNN to classify the input sample images into normal image or abnormal image. The classification accuracy of the Hybrid classifier SVM-KNN gives better accuracy rate than SVM classifier.

4. R. Usha [4] ,CBIR is done by combining the two features such as color and texture. The texture feature are extracted by segmentation based fractal texture analysis. Color features are extracted by using HSV histogram. The main focus of this work is classification of image using SVM algorithm.

5. Mohanapriya.S [5],this paper introduce a novel method of using SVM classifier followed by KNN for CBIR using shape feature and texture feature of MRI brain image. The Gray level cooccurance matrix are used to extract texture feature of images. The classification is performed on the dataset and its classified into three categories.

\section{METHODOLOGY}

The effective image processing techniques are achieved by extracting the features such as shape and texture from an image in CBIR system. The figure 1 describes the basic functionality of content based image retrieval.

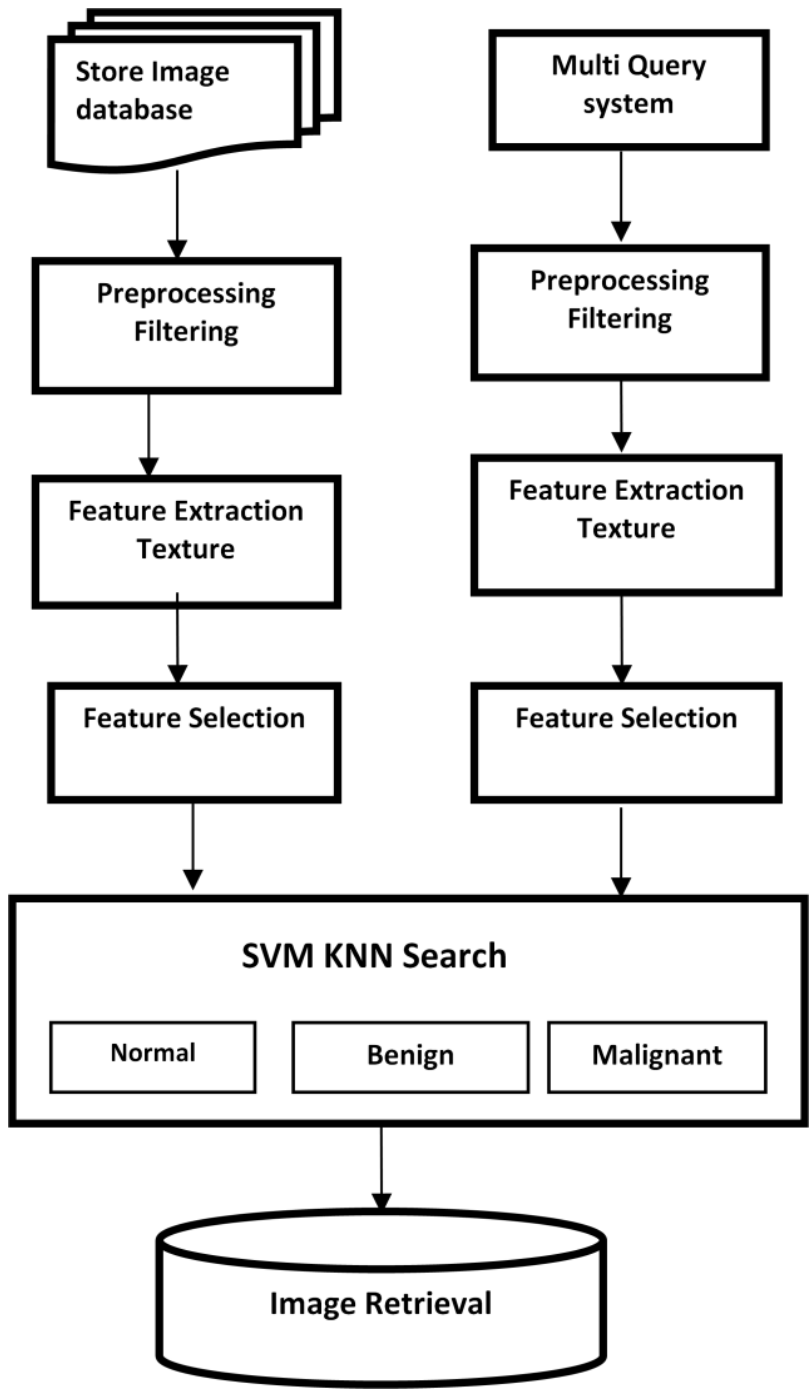

Figure 1

\section{FEATURE EXTRACTION}

Similarity measurement criteria, query formulation, choosing more discriminative features and so on these are many factors affecting the performance and accuracy of CBIR system. The designed CBIR system performed efficiently, the first step is to extract the discriminative feature from the images in the reference database. These features will also be calculated for query images. The most important discriminating characteristics of mri brain tumor images is gray scale the preprocessing is performed to removal $\mathrm{f}$ noise because MRI images are degraded by different kind of noises. It is very important to have good quality of images for accurate observations

\section{Texture Feature}

The extraction of the texture feature is done by segmentation based Fractal texture analysis .Accurately capture the textural characteristics of an image ,there are various texture analysis algorithms are used such as filter banks or gray level cooccurrence matrix have to consider multiple orientation and scales. The computational cost for applying this method may be heavy,SFTA works much faster in terms of extracting the texture feature of image, when compared to Gabor and Haralick methods. The main objective of using SFTA is to extract texture feature in an image which results in the formation of vector[6].This Extraction algorithm creates the binary images by decomposing the input image which the fractal dimensions of the regions' borders are computed from to describe the segmented texture patterns.

\section{Feature Selection}

Feature selection algorithms are very important to recognition and classification system. There are many automatic feature selection methods, Branch and bound is not of the most traditional algorithm, which is an optimal method for monotonic feature set. But this algorithm has some limitations for large feature space, it does an exhaustive search and its time complexity is exponential on the size of the feature space. In this worked we have used Sequential forward selection (SFS) algorithm select the discriminative features among the feature vector.SFS is a simple is the simplest greedy search algorithm.

\section{TWO TYRE RETRIEVAL}

\section{FIRST TYRE: Classification of tumor types with SVM.}

Support vector machine (SVM) is a accurate learning techniques and powerful supervised classifier that has been introduced in 1995.

SVM has successful classification results in various application domains such as medical diagnosis. The classifying ability of SVM is better than other pattern recognition methods, in various application still exist some problems such as complex application has low classifying accuracy and difficulty in choosing the kernel function parameters. To solve these problem, a simple and effective improved SVM classifying algorithm was proposed by Li et al. (2002), which combines SVM with the K-nearest neighbor $(\mathrm{KNN})$ classifier. is a pattern recognition algorithm which classify brain MRI images under various categories which refers to brain tumor.SVM classifier are used to classify the images using feature vector gained from the MRI images. A support vector machine search an optimal separating hyperplane between members and non-members of a given class in a high dimension feature space. In data preprocessing step filtering has done to reduce the noise from images and 
features are extracted using SFTA method. In this method the two classes are normal or abnormal brain. The maximum margin classifier is the basic principal of the support vector machine.

\section{TWO TIRE: Classification of query}

This concept is used in pattern recognition for classifying objects based on the closest training examples in a feature space. This algorithm is only worked for classification, where objects are classified by a more number of votes of its neighbor. If $\mathrm{k}=1$, the objects is consideration is assigned the class of its nearest neighbor. The algorithm has a classification phase and training phase. The feature vectors and class labels of the training samples. The classes with the more frequent samples have a tendency to control the prediction of the new vector, because they tend to come up in the k-nearest neighbor when neighbors are computed due to their large samples this is the one of the drawback of it. Ecludian distance are used for the similarity comparison. The choice of $\mathrm{k}$ depends on the data, reduced the effect of the noise on the classification by selecting the larger value of $\mathrm{k}$.

For similarity comparison, Euclidian distance is used, d followed by.

$$
D 2(a 1, a 2)=\sqrt{ } \sum_{j=1}^{k}(a 1, j-a 2, j) 2
$$

Here $\mathrm{n}$ refers to the number of images in the database.

To calculate each database image and query image to find weighted score in relevant image.

\section{CONCLUSION}

CBIR is done by using texture and shape feature extraction introducing a method of support vector machine(SVM) classifier followed by K-nearest neighbor (K-NN).In this paper propose a efficient retrieval of the MR images using a supervised classifier which concentrates on the extracted feature. Segmentation based fractal texture analysis(SFTA) algorithm is used to extract the texture feature from images .Reduce the dimensionality of data ,select the best feature from the extracted feature to train the classifier. Its automatically reduces the computation cost. The extracted feature are classified into three categories such as normal, benign and malignant by performing the classification on the dataset. The query image is classified by the classifier to a particular class and relevant images are retrieved from the database. To improve the accuracy, calculate the precision value and recall in relevant images.

\section{REFERENCES}

[1] Guoyong Duan, Jing Yang, Yilong Yang, "Content based image retrieval research" ,2011 International conferances on physics science and technology(ICPST 2011)

[2] Priyanka P. Buch,Madhuri V. Vaghasia, Sahista M. Machchhar,"Comparative analysis of content based image retrieval using both color and texture", Institute of technology, Nirma univercity, Ahemedabad-382 481, December,2011.

[3] Keatn Machhale, Hari babu nandpur, Vivek kapur, Laxmi Kostan, "MRI brain cancer classification using hybrid classifier(SVM-KNN)," 2015 International conference on industrial instrumentation and control(ICIC),May-2015.

[4] R. Usha, K. Perumal,"Content based image retrieval using combined features of color and texture features with SVM classification", International journal of computer science and communication networks, $\mathrm{Vol} 4(5)$, $169-174.1$

[5] Mohanapriya.S, Vadivel.M," Automatic retrieval of MRI brain image using multiqueries system", IEEE, 2014

[6] Alceu Ferraz Costa, Gabriel Humpire-Mamani, Agma Juci Machado Traina" An Efficient Algorithm for Fractal Analysis of Textures", 2012 XXV SIBGRAPI Conference on Graphics, Patterns and Images

[7] H. Muller, N. Michoux, D. Bandon, and A. Geissbuhler, "A review of content-based image retrieval systems in medical applications clinical benefits and future directions," Int. J. Med. Informat., vol. 73, no.1pp. 1-23, 2004.

[8] H.B.Kekre, Tanuja K. Sarode, Sudeep D. Thepade, Vaishali Suryavanshi,'Improved texture feature based image trtrieval using Kekre,s fast codebook generation algorithm",August 2011. 